\title{
Les groupes de PME, une structure particulière pour la communication en entreprise
}

\author{
Alexandre Mallard
}

\section{(2) OpenEdition}

1 Journals

Édition électronique

URL : http://journals.openedition.org/communicationorganisation/2955

DOI : 10.4000/communicationorganisation.2955

ISSN : $1775-3546$

Éditeur

Presses universitaires de Bordeaux

Édition imprimée

Date de publication : 2 octobre 2004

ISSN : 1168-5549

Référence électronique

Alexandre Mallard, «Les groupes de PME, une structure particulière pour la communication en entreprise », Communication et organisation [En ligne], 25 | 2004, mis en ligne le 27 mars 2012, consulté le 01 mai 2019. URL : http://journals.openedition.org/communicationorganisation/2955 ; DOI : 10.4000/communicationorganisation.2955

Ce document a été généré automatiquement le 1 mai 2019.

(c) Presses universitaires de Bordeaux 


\title{
Les groupes de PME, une structure particulière pour la communication en entreprise
}

\author{
Alexandre Mallard
}

\section{Introduction}

1 La tendance des entreprises à se regrouper pour agir dans l'environnement économique constitue un sujet qui a donné lieu à de nombreux travaux au cours des dernières années. Ce que l'on met derrière le terme vague d'entreprise en réseau renvoie à des réalités très diverses (Castells, 1998). Une des acceptions dominantes de ce terme concerne sans doute le développement massif de la sous-traitance et la décomposition associée des chaînes de valeurs dans un tissu industriel qui a connu au cours des 50 dernières années plusieurs mouvements de recompositions structurelles et un processus d'internationalisation marqué. Une autre réalité industrielle associée à cette notion concerne l'extension progressive des démarches de mise en commun de ressources dans différents domaines de l'activité productive : franchise, groupement d'achat, réseau de conseil, joint venture, coopérations de recherche.. On pense parfois également au développement de pratiques coopératives en matière de conception de produits et de services, émergent dans certains domaines d'activités. Au total, ces modes de regroupement conduisent à des collectifs productifs inédits qui posent d'ailleurs de sérieuses questions au travail de mesure statistique, qui se doit d'inventer de nouveaux concepts et de nouvelles méthodes pour en cerner le périmètre et la nature (INSEE, 1997).

Le regroupement d'entreprises attire encore plus l'attention lorsque l'on parle de PME, peut-être parce qu'il y est perçu comme une façon de contrebalancer les effets de taille sur le marché, et de renforcer la capacité des petites entreprises à se positionner dans des milieux économiques favorables aux grandes entreprises intégrées et aux grands groupes - même si le paramètre taille joue un rôle ambivalent pour appréhender les spécificités de gestion que l'on peut attribuer à la PME (Torrès, 1998; Harisson, 1995 ; Marchesnay, 
2003). Plus généralement, le regroupement apparaît comme une ressource pour survivre et se développer dans un environnement marqué par l'exacerbation de la concurrence, l'ouverture des marchés et la mondialisation, la montée d'une économie de la variété qui suppose de rechercher des coopérations pour élargir les offres et les adapter aux exigences de consommateurs dépeints comme toujours plus exigeants.

Les modalités de regroupement entre PME ont fait l'objet de recherches diverses. Certains analystes, voient par exemple dans la diffusion des technologies de l'information et de la communication un levier de transformation de l'économie débouchant sur de nouvelles formes d'association entre des entreprises de petites tailles. Il existe ainsi toute une littérature, qui dispose aujourd'hui d'une forte visibilité, et qui associe le développement des nouvelles technologies à un processus de désintermédiation généralisée qui entrainerait de façon structurelle une situation dans laquelle la coopération entre PME serait la règle d'organisation de l'univers productif (Laubacher et Malone, 2001). Les sciences économiques et de gestion se sont intéressées, plus anciennement et de façon plus traditionnelle, aux diverses modalités du regroupement de PME. La montée des alliances stratégiques et des accords de coopération entre PME est un des aspects du phénomène qu'ils ont bien identifié (Puthod, 1998; Menguzzato-Boulard, 2003). Les districts industriels constituent une autre forme de coordination entre PME, qui a beaucoup excité la curiosité des chercheurs (Beccatini, 1992; Piore et Sabel, 1994). Le développement un peu plus récent de recherches autour d'une de leurs variantes, les systèmes productifs locaux (Crouch et al, 2001 ; Courault, 2000), n'en dément pas l'intérêt, comme en témoigne d'ailleurs ce numéro de communication et organisation qui leur est consacré.

Dans le cadre de cet article, on voudrait centrer l'attention sur un autre mode de regroupement entre PME (qui recoupe parfois ceux que l'on vient de présenter) et qui concerne le développement des structures de groupes à proprement parler. Un certain nombre de recherches récentes, réalisées par des spécialistes de statistique économique et gestion, ont signalé l'importance du développement de ces groupes de PME au cours des 20 dernières années. Les entités en question sont désignées parfois par le terme de micro-groupe, parfois par celui d'hypogroupe. On peut se demander quelle est la nature de ce qui se présente comme un mouvement de concentration financière et capitalistique croissant dans le secteur des petites entreprises. Par ailleurs, les recherches consacrées aux usages des technologies d'information et de communication dans les PME (Boutary et al, 2003 ; Dhaleine et al, 1999 ; Gadille et d'Iribarne, 2000) n'ont pas véritablement abordé la question des groupes. On se fixera ici comme objectif de dresser un état des lieux des recherches sur les groupes de PME, et de formuler quelques propositions sommaires pour une analyse des modalités de la communication et de la circulation de l'information dans ces structures un peu particulières. On procédera en trois temps : on tentera tout d'abord de resituer l'apparition des PME et des groupes dans le temps long de l'histoire industrielle depuis le début du siècle dernier; on proposera ensuite une synthèse de données qualitatives et quantitatives sur le phénomène des micro-groupes; pour finir, on présentera quelques exemples issus d'une enquête sur les systèmes de communication dans les groupes de PME, afin d'illustrer les voies possibles d'une réflexion sur la communication dans ce type de structures. 


\section{Grandes entreprises, PME, groupes : des entités productives interdépendantes et liées par une histoires commune}

\section{Des origines de l'industrie moderne au modèle fordien-taylorien}

L'intérêt même que suscitent les phénomènes de groupement de PME pour divers analystes comporte un risque : celui de laisser penser que notre période actuelle verrait l'apparition de formes productives nouvelles par combinaison de structures élémentaires formant la base du tissu économique. Nous assisterions à un processus de regroupement d'unités économiques qui jusqu'ici seraient restées atomisées et qui, de par l'apparition de conditions économiques, techniques ou juridiques inédites, pourraient désormais s'associer entre elles pour former des unités de taille plus grande. Cette image comporte sans doute une part de vérité pour autant qu'on accepte de partir de la forme PME comme un point de départ. Il parait néanmoins raisonnable de ne pas s'en tenir là, et d'intégrer cette analyse dans le cadre d'une réflexion sur l'histoire des liens entre entreprises de grande et de petite (ou moyenne) taille. On est ainsi conduit à retourner la logique et à considérer, au moins momentanément, la PME comme un point d'arrivée dans l'histoire industrielle. Le panorama historique et analytique que proposent Trouvé et Courault (2000) est ici très instructif. Ils distinguent 3 périodes dans l'histoire des formes productives : l'ère pré-industrielle, le régime fordien-taylorien et le régime post-fordien.

6 Dans la première période, la petite entreprise est bien la base de l'économie et apparaît repérable sous deux figures différentes. La première correspond à un modèle familial, dans lequel la production s'enracine dans des relations fondées sur le domestique. Cette forme de production «qui consiste en de minuscules ateliers familiaux disposés en nébuleuses » va se trouver progressivement dominée par une seconde forme "qui voit entrer en scène les premiers entrepreneurs que sont les marchands ». Les marchands vont être historiquement les premiers à occuper la place du donneur d'ordre, et à organiser la sous-traitance de biens qu'ils s'occuperont ensuite de distribuer. La seconde période, celle du régime fordien-taylorien, est précisément caractérisée par l'apparition et le triomphe de la grande entreprise. Elle constitue la forme d'organisation résultant naturellement du processus de concentration de l'appareil productif que connaissent les sociétés modernes depuis le début $\mathrm{du} x \mathrm{x}^{\mathrm{ème}}$ siècle, un processus qui n'a pas toujours été autonome et qui a connu dans certains pays - c'est le cas en France - le soutien fort de la puissance publique. Dès lors, les petites entreprises ont pu apparaître comme périphériques dans ce modèle industriel, alors mêmes qu'elles continuaient naturellement à exister sous des formes très diverses, marquées globalement par une opposition entre deux types de structures : d'un côté la petite société indépendante, gérée par le dirigeant-propriétaire ou un cercle de famille élargi, de l'autre des petites entreprises qui tendent à être contrôlées par les grandes, soit par la mise en place de relations de sous-traitance entraînant une forte dépendance, soit par le biais des rachats, fusions et absorptions. ${ }^{1}$ 


\section{La période post-fordienne}

7 C'est dans la troisième période, dite " post-fordienne » que la PME va peu à peu acquérir un statut proche de celui qu'on lui connaît aujourd'hui. Cette troisième période est marquée par des transformations macro-économiques d'ensemble qui introduisent une rupture forte par rapport à la précédente. Elle connaît la montée d'une économie de la variété, une intensification de la concurrence et l'émergence de facteurs de compétitivité nouveaux (qualité, délais, variétés, réactivité, innovation..) par rapport à ceux de la production standardisée et de la consommation de masse. Sur le plan de l'organisation productive, elle correspond à l'émergence d'un paradigme de la souplesse, à l'épuisement des structures hiérarchisées et concentrées de la période précédente, des phénomènes qui mèneront à ce que l'on appellera notamment la désintégration verticale : les grandes entreprises externalisent une partie des ressources qu'elles avaient constitué en leur sein, de façon à gérer certains segments du processus productif avec la flexibilité que permet le recours au marché. Enfin, sur le plan de la régulation sociale, la période post-fordienne témoigne d'une crise des relations professionnelles et d'une flexibilisation progressive du rapport salarial.

8 À la différence du régime précédent, les PME ont été perçues dans ce régime comme porteuses d'un rôle économique spécifique. Elles ont été valorisées pour leur souplesse et leur capacité d'adaptation à un environnement économique perçu comme plus fluctuant. On en a attendu une capacité à créer des emplois en période de crise, une capacité qui se sera vue démentie par les analyses ultérieure. ${ }^{2}$ Au final, la contribution des PME au fonctionnement de ce modèle peut être perçue au travers de deux modèles distincts. La « firme en réseau » constitue un premier modèle, même si ces organisations en réseaux peuvent correspondre à des formes de coordinations économiques contrastées : réseaux verticaux pour des systèmes industriels structurés autour de relations de sous-traitance qui se sont accrues, réseaux horizontaux pour des configurations relationnelles moins polarisées par la domination de certains acteurs, mobilisant plus fréquemment des modes de coopération. Les petites entreprises traditionnelles, artisanales, familiales ou indépendantes représentent un deuxième modèle de PME qui affirme sa persistance dans une économie gagnée par la montée des réseaux.

\section{Le développement des groupes}

La multiplication des groupes dans l'industrie Française au cours des 25 dernières années constitue peut-être une manifestation parmi d'autres du changement de régime productif intervenu dans le prolongement du post-fordisme - même si les groupes en eux-mêmes ont une existence bien plus ancienne. Cette multiplication est bien repérée dans les études de statistique économique, qui indiquent ainsi que les groupes passent de 1300 en 1980 à 6700 en 1995 pour atteindre un chiffre d'environ 12000 en 2003 (Vergeau et Chabanas, 1997 ; INSEE, 2004). Plus d'un tiers de l'emploi dans les PME se fait désormais dans des entreprises appartenant à des groupes. La présence des groupes est marquée par des inégalités sectorielles: ils prédominent dans les secteurs fortement capitalistiques comme l'industrie automobile, l'énergie et les activités financières; ils contribuent à structurer fortement l'industrie française (Feuvrier, 2000); ils sont plus faiblement représentés dans la construction, les services aux particuliers, le commerce. Au total, les groupes employaient plus de 4 millions de salariés en 1981, ils en emploieraient le double 
en 2003, produisant désormais $60 \%$ de la valeur ajoutée de l'économie française (INSEE, 2004 ; Skalitz, 2002)

Les dynamiques qui mènent à sa constitution ont fait l'objet de recherche (Jacquemin, 1989). Pour une entreprise donnée, la structuration en groupe intervient en généra ! dans des conditions qui rendent une mutation nécessaire, soit du fait de processus de réorganisation, soit du fait de pressions externes sur l'entreprise. Elle s'intègre parfois dans une stratégie de croissance qui peut prendre plusieurs aspects. Il peut s'agir de s'attaquer à un plus grand marché, de mieux contrôler le circuit commercial en aval de l'activité pour garantir l'écoulement des produits, d'exercer un contrôle de l'amont pour garantir les approvisionnements, ou de diversifier ses activités. La structure de groupe procure alors de multiples bénéfices. Elle constitue tout d'abord une réponse nécessaire lorsque l'activité s'exerce sur différents territoires nationaux, pour s'adapter aux conditions réglementaires, politiques, institutionnelles de chaque pays. Elle rend ses opérations de croissance relativement réversibles, puisque la revente de participations est une opération plus simple que le démembrement d'une partie de l'activité interne à une firme. Le groupe permet également, de par l'autonomie juridique des sociétés, de réduire les risques financiers et fournit une structure de responsabilisation de la gestion qui est souvent plus efficace que pour des activités organisées en départements dans une même société.

Mais la constitution de groupes à partir de grandes entreprises peut se faire également en sens inverse de celui de la croissance, à partir de la décomposition d'une activité économique unitaire en sous-éléments plus autonome. La filialisation des activités donne naissance à des entreprises plus petites, et souvent à des PME. La constitution de groupes comportant des PME satellites autour de la grande entreprise cœur désigne une forme parmi d'autres (cession et essaimage des activités jugées non stratégiques, franchises, sous-traitances fortes en contrat d'exclusivité avec des PME indépendantes..) du processus de «PMI-sation» de l'appareil productif apparu depuis les années 80 avec le mouvement de désintégration verticale (Trouvé et Courault, 2000).

$\mathrm{Au}$ total, le groupe constitue dans tous les cas une structure qui permet d'allier la possibilité de concentration des ressources et la décentralisation de la gestion et des responsabilités. Même si le groupement d'entreprise ne constitue pas une forme de la relation inter-entreprise au sens classique du terme (Baudry, 1995), il est possible de le positionner dans le cadre de la réflexion sur l'articulation entre coordination par l'organisation et coordination par le marché. Jacquemin la place ainsi au nombre des formes intermédiaires qui «sont susceptibles, dans certaines conditions, d'à la fois réduire les coûts de transaction qui auraient été encourus si celles-ci s'étaient effectuées via le marché, et d'éviter certains coûts d'organisation qui se seraient manifestés si la transaction avait été totalement internalisée au sein de la firme. » (Jacquemin, 1989, p.8). Les échanges de ressources à l'intérieur d'un groupe se font en général à des prix inférieurs à ceux du marché. La structure permet également la mise en commun de fonctions transverses : la comptabilité, la R\&D, la gestion du personnel, etc. Il devient possible aux entités membres (les filiales) d'exercer des activités peu rentables tout en bénéficiant de la protection de l'ensemble de la structure du groupe. 


\section{Des groupes de PME}

\section{L'émergence des micro-groupes}

13 deux dynamiques très intéressantes pour notre perspective. La première est relative à l'éclatement des grands groupes, consécutif du processus que l'on vient d'évoquer. Entre 1990 et 1995 par exemple, le nombre de filiales des grands groupes passe de 3000 à 10300 . Le nombre moyen d'entreprises composant ces grands groupes passe durant cette période de 40 en 1980 à 75 en 1989 et à 125 en 1995 (Vergeau et Chabanas, 1997). On voit bien ici un des effets du processus de désintégration verticale, puisque ces chiffres traduisent largement la tendance des grandes entreprises à filialiser certaines parties de leurs activités. Mais c'est surtout la deuxième tendance, celle de l'explosion des micro-groupes, qui explique le phénomène d'ensemble.

Image1

Figure 1 : la présence des groupes dans l'industrie Française selon l'enquête LIFI (d'après Vergeau et Chabanas, 1997 ; Loiseau, 2001 ; INSEE, 2004)

On désigne par le terme de micro-groupe des regroupements de PME dont la taille totale n'excède pas 500 salariés. La figure 1 montre l'évolution du nombre de groupes et de micro-groupes dans l'économie Française tels qu'on peut les repérer d'après l'enquête Liaisons Financières (LIFI) de l'INSEE. ${ }^{3}$ Un micro-groupe rassemble en autour de 4 entreprises. Une PME sur 4 appartient à un groupe, et une sur 8 appartient à un microgroupe (données relatives à l'année 1998). Il semble que le processus de constitution d'entités micro-groupes puisse relever de deux logiques différentes, qui peuvent parfois être associées mais qui dans leur principe sont clairement distinctes. Dans la première logique, la structure de groupe est un des résultats du rassemblement de plusieurs activités ou entreprises différentes, par le biais de rachat ou de prise de participation financière dans le capital d'une autre entreprise. Dans la seconde, le groupe résulte d'une réorganisation de la structure de propriété d'une entreprise. A priori, la première logique a des incidences organisationnelles et économiques, tandis que la seconde correspond à une réalité essentiellement juridique. Le processus de regroupement a été analysé dans le cadre des recherches sur la PME et l'entrepreneuriat, sous la thématique de «l'hypogroupement » (Marchesnay, 1991). Ces recherches proposent une compréhension qualitative du processus en le situant dans la visée d'une analyse stratégique.

\section{Une analyse stratégique qualitative du groupement pour les petites entreprises}

Dans cette tradition, l'hypogroupe est défini « comme un groupe de petites entreprises unies entre elles par des relations financières donnant à l'une d'entre elles (la holding) le pouvoir de décision sur les autres (les filiales) » (Debray et Leyronas, 1998) Ce courant de recherches insiste sur la nécessité, pour comprendre le processus d'hypogroupement, de dépasser les analyses du processus de croissance organisationnelle qui l'envisagent comme une évolution « naturelle » de la PME vers le modèle de la grande entreprise, avec les transformations du style de management et de l'organisation qu'elles sous-tendent. Il

Communication et organisation, 25 | 2004 
met l'accent au contraire sur les spécificités de la petite entreprise : l'importance du rôle du dirigeant-propriétaire, l'organisation simplifiée, le caractère relativement spécialisé des compétences et de l'activité, l'imprégnation forte dans l'environnement.

Dans cette perspective, la constitution de groupe de PME fait partie des stratégies de croissance hors taille, c'est-à-dire des stratégies qui permettent aux petites entreprises d'assurer leur développement sans pour autant souffrir de deux séries d'inconvénients symétriques: les inconvénients liés au développement d'activités externes (soustraitance, prestations externalisées), qui comportent des risques de mise en dépendance des partenaires ou de perte d'avantages compétitifs; les inconvénients liés à l'augmentation de taille en interne, comme la rigidification des procédures, la perte de contrôle ou de maitrise des transactions internes, la déperdition d'informations, etc. L'organisation et la répartition de la propriété entre les actionnaires des différentes filiales va fournir non seulement des possibilités de jeu dans les ressources financières (par exemple ouverture du capital des filiales à des tiers minoritaires) et, en contrepartie, des jeux spécifiques dans l'exercice du contrôle décisionnel, de par l'exigence plus ou moins prégnante d'intégrer les attentes des actionnaires dans la gestion.

Sur cette base, Debray (2000) est conduite à distinguer deux types de logiques d'hypogroupement. On a d'un côté la logique d'action patrimoniale, qui s'assimile à un processus d'accumulation d'actifs et de savoir-faire sur le mode de la constitution d'un stock. Elle sous-tend souvent des objectifs de pérennisation de l'activité et de transmission à des héritiers ou à d'autres membres de la famille, et le capital pourra faire l'objet de verrouillage de façon à conserver au dirigeant l'intégralité du pouvoir décisionnel. De l'autre côté, la logique entrepreneuriale intègre plus largement des choix industriels liés à l'activité. Il s'agit de valoriser des actifs au travers d'une stratégie de développement, bien souvent avec une logique d'activités complémentaires entre les diverses filiales du groupe. La gestion de la propriété au sein du groupe sera plus ouverte, le dirigeant étant prêt à sacrifier une partie de son indépendance dans une visée de développement, tout en conservant la possibilité d'arbitrer des décisions stratégiques. $\mathrm{Si}$ le premier groupe reste proche, dans ses modes de gestion, de la PME traditionnelle, il se rapproche dans le second cas de la grande entreprise.

\section{Une typologie des micro-groupes}

Ces analyses qualitatives d'ordre stratégique recoupent globalement les conclusions des études quantitatives qui ont constaté le phénomène statistique dans toute son ampleur. À partir des données de l'enquête LIFI, Loiseau (2001) propose une analyse des motivations dans la constitution des groupes et une typologie des groupes de PME. Il identifie pour sa part trois catégories de motivations. La première renvoie au développement : l'achat et la prise de participation dans d'autres entreprises prennent sens dans une logique de croissance, d'implantations dans de nouvelles régions ou de développement de nouvelles activité. La structure de groupe présente l'avantage de «cantonner les risques, par rapport à une croissance interne à l'entreprise, puisque la structure groupe permet de se séparer d'une entreprise en difficulté sans engager la responsabilité de toutes les entreprises du groupe.» (Loiseau, 2001). La seconde motivation est celle de la rationalisation des activités : des PME parvenues à une taille données trouvent certains intérêts à répartir leurs activités dans des structures juridiques plus petite. La dernière motivation concerne la transmission du patrimoine. Loiseau note ainsi que parmi les 
microgroupes créés entre 1990 et 1998, un sur 5 était composé d'une holding, tête du groupe, et d'une seule filiale. La structure de holding-entreprise familiale dispose en France d'une fiscalité allégée qui la rend attractive lors d'opérations de succession ou de donation dans certaines situations. En outre, la forme holding constitue une solution pratique pour conserver une unité de direction à une entreprise même lorsque le capital est divisé entre plusieurs héritiers. Enfin, un autre phénomène semble avoir joué un rôle important: l'internationalisation de l'économie: profitant de dispositions légales incitatives au niveau français et communautaires de nombreux groupes étrangers sont venus s'implanter sur le territoire français depuis le début des années 1990 par le biais du rachat d'une entreprise existante.

19 Dans l'ensemble, l'analyse statistique suggère que les micro-groupes représentent une réalité économique relativement homogène. Notant qu'un tiers d'entre eux n'est composé que de 2 entités et $80 \%$ d'au plus 5 entreprises, Loiseau (2001) propose d'en distinguer 4 types:

20 - les micro-groupes entreprises. Ils sont constitués de deux entreprises dont la tête est une holding et dont la seule activité réelle relève de l'entretreprise contrôlée. Ils sont présents dans les secteurs industriels (46\%) et dans le commerce (33\%). À première vue, on trouve dans cette structure beaucoup d'entreprises à gestion familiale structurées en groupes pour des raisons patrimoniales, sans véritablement qu'il y ait plusieurs entités productives en interaction.

21 - les micro-groupes monovalents. Ils comportent plusieurs entreprises, dont l'activité s'exerce dans le même domaine. Implantés dans l'industrie (33\%), le commerce (26\%), « ils correspondent surtout à des entreprises qui ont soit rationalisé leur organisation, soit recherché une croissance externe (création ou rachat) 》 (Loiseau, 2001).

22 - Les micro-groupes bivalents. Ils rassemblent des activités qui s'exercent dans deux domaines différents. Une partie d'entre eux résulte d'une rationalisation de l'organisation financière d'un ensemble d'entreprise: ils peuvent ainsi avoir créé une société immobilière qui gère les locaux du groupe. Une autre partie correspond à des groupes qui ont diversifié leur activité en créant par exemple une société commerciale ou de service en destinée à soutenir l'activité principale.

23 On y trouve les associations d'activités suivantes : industrielles et commerciales (18\%), industrielles et services aux entreprises (16\%), commerciales et services aux entreprises (11\%), commerciales et immobilières (10\%), industrielles et immobilières (9\%).

24 - Les micro-groupes polyvalents. Ce sont des entités complexes qui combinent la rationalisation de l'organisation productive et l'optimisation financière. Ils sont implantés dans trois domaines ou plus. Tout comme pour les groupes bivalents, une activité est centrale tandis que les autres activités sont périphériques, et destinée à la soutenir.

Image 2

Tableau 1 : part et taille moyenne des différents types de micro-groupes en 1998 (d'après Loiseau, 2001) 


\section{Communiquer dans des groupes de PME ? Quelques pistes de réflexion}

Nous voudrions pour finir amorcer une réflexion sur les modalités d'usage des systèmes d'information et de communication dans les groupes de PME. Nous présenterons pour cela quelques exemples de groupes de PME dont nous avons pu interroger les dirigeants à l'occasion d'une enquête menée à France Télécom R\&D à la fin de l'année 2002. Cette enquête partait de l'idée que les pratiques de communication et de coopération dans ce type de structures pouvaient receler des particularités intéressantes, dues précisément au besoin de faire circuler l'information entre des collectifs de travail différenciés. Les 21 PME retenues pour cette enquête ${ }^{4}$ qualitative avaient été choisies pour leur sensibilité a priori à la problématique de la communication et de la coopération à distance, dans une même structure (entreprises ou groupe multi-sites) ou dans une structure différente (sociétés travaillant en réseau avec leurs clients et leurs partenaires). Dans chacune de ces entreprises, on a mené un entretien approfondi d'une à deux heures avec le chef d'entreprise ou le responsable informatique, en cherchant à qualifier les équipements en technologies de communication et, tant que possible, les usages organisationnels associés. Il s'est avéré a posteriori que 14 de ces entreprises appartenaient à des groupes (qui étaient dans tous les cas des groupes de PME). L'étude nous a largement convaincu de la complexité du phénomène, une complexité que nous nous proposons d'illustrer ici en exposant brièvement le cas de 3 de ces groupes.

\section{Martin Leclair : des systèmes de communication partiellement cohérents pour un groupe monovalent à caractère patrimonial}

Le premier groupe, que nous appellerons Martin Leclair ${ }^{5}$, exerce une activité dans le domaine des articles d'éclairage. II se compose de trois sociétés qui occupent des positions complémentaires dans ce domaine. La société mère est située à Paris et emploie 65 personnes. Elle exerce une activité exclusivement commerciale, de commerce de gros et de distribution. Il existe deux filiales, une à Vitry ( 25 personnes) et une en Italie (50 personnes), qui assurent la fabrication des articles et une partie de leur distribution, sur des créneaux qui ne se correspondent pas complètement : la filiale de Vitry ne réalise que des lampes, tandis que la filiale Italienne est activée sur l'ensemble des articles d'éclairage. Le chef de l'entreprise est âgé d'une cinquantaine d'année et présente en quelque sorte un profil de patron consciencieux et affairé. Son bureau se trouve dans des locaux situés dans le 4ème arrondissement, dans une ancienne manufacture parisienne dans ce quartier du faubourg Saint Antoine où proliféraient les artisans et entreprises du mobilier.

Le groupe est issu de 2 rachats, en 1996 et 2001, qui ont été réalisés par opportunité et également par le souci de sécuriser une partie de l'approvisionnement («nous aurions perdu la fabrication de nos produits, et les usines auraient pu partir chez des concurrents »), et donc d'étendre un peu son assise sur la chaîne de fabrication. Le degré d'intégration entre ces trois firmes est moyen, principalement de nature entrepreneurial, avec des transactions croisées: Paris distribue une partie des produits italiens ( $25 \% \mathrm{du}$ marché) et de Vitry. En revanche, aucune identité commerciale commune n'est affichée les clients ne savent pas en général que les trois sociétés relèvent d'un même groupement 
- et il semble que la structure de groupe ne soit que faiblement le support de logiques de coopération transverse entre les services des différentes filiales.

La gestion des systèmes d'information et de communication répond à ce schéma de cohérence parcellaire. L'équipement en téléphonie des trois sociétés est géré de façon indépendante. Il en est autrement pour l'informatique de gestion, qui s'organise autour d'un système d'information centralisé destiné à assurer l'ensemble du processus de gestion commerciale, et intégrant notamment les applicatifs EDI permettant d'interagir avec les clients de la grande distribution, avec lesquels sont concentrés entre 30 et $40 \%$ de l'activité. Sur ce plan, les sociétés de Paris et Vitry ont procédé à une interconnexion des systèmes d'information et à un partage de base informatique. Pour autant, la discussion avec le dirigeant suggère que c'est plus une opportunité d'économie ponctuelle qu'une volonté stratégique qui a guidé cette mise en commun : «Quand on a racheté l'entreprise, on n'a pas voulu racheter de système informatique alors on les a interconnecté. » De la même façon, aucune interconnexion n'a été réalisée avec la filiale Italienne. De façon générale, le dirigeant nous laissera l'impression que la « révolution internet » n'est pas encore arrivée dans cette activité, voire qu'elle inspire une certaine méfiance, et que la mise en œuvre d'une démarche d'ensemble au niveau du groupe sur le versant de la communication n'est pas à l'ordre du jour.

\section{Publi-Factory : un groupe polyvalent et international, à caractère managérial et visant une stratégie globale pour son infrastructure de communication}

29 Changement de décor pour le groupe Publi-factory, actif dans les domaines du conseil en publicité et du "publishing ». Le métier du publishing comprend tous les services qui démarrent après la phase de créativité en matière de publicité et qui s'arrêtent à la livraison de supports chez le client. Un grand groupe de distribution confie ainsi la fabrication de son catalogue à Publi-Factory, qui en assure la conception et la livraison, la fabrication seule étant sous-traitée. Publi-Eactory constitue un groupe de communication actif à l'échelon européen. En France, il rassemble une série d'entreprises articulant les différentes activités de la chaîne de traitement: le groupe comprend sur le versant français une société chargée de faire de la gestion déléguée de l'édition publicitaire, une entreprise de pré-presse, une centrale d'achat pour le papier, une entreprise de transport, une filiale centrée sur la communication sur les lieux de vente. Le groupe a développé des implantations dans plusieurs pays européens, entre autres par une politique de rachat de petites agences nationales depuis le début des années 90 : par exemple, la filiale suisse acquise en 2000 comporte 30 personnes, la filiale italienne comprend 3 agences, la filiale anglaise emploie 15 personnes issues d'une agence créée en 1991 et rattachée au groupe en 2001, etc. Au total, on a affaire à un groupe extrêmement intégré, aux niveaux stratégique, gestionnaire, financier et organisationnels. Comme il se doit dans l'univers de la communication, ce caractère intégré est publiquement affiché, par exemple sur le somptueux site web de la société qui permet de prendre connaissance de sa composition, de sa stratégie, et des différents points de contact.

30 En matière de système d'information, le groupe vise une cohérence globale en prise directe avec cette configuration intégrée. Les télécommunications et l'infrastructure du système d'information sont gérées en central pour tout le groupe. Elles jouent un rôle bien plus primordial, il est vrai, que dans le cas précédent du négociant en article 
d'éclairages, de par l'importance que prend l'informatique dans le métier. Ainsi à Montrouge, l'implantation de la société principale du groupe en France, le parc informatique se compose de plus d'environ 250 ordinateurs personnels (partiellement macintosh, partiellement $\mathrm{PC}$ ) en réseau. Un service d'échange de données très haut débit relie les différentes entreprises du groupe, de façon à permettre la circulation des documents numériques qui forment un des supports principaux de l'activité. Le site de Montrouge met à disposition plus de 200 lignes téléphoniques fixes et 80 téléphones mobiles pour certains collaborateurs. Au-delà de l'importance des services de télécommunication pour l'activité, que traduisent ces chiffres, c'est son mode de gestion qui frappe, par contraste également avec le cas précédent. Un des moteurs de la gestion concerne la réduction des coûts par le choix des meilleures solutions au niveau européen. Les différents sites européens ne sont pas encore vraiment reliés entre eux, et la question de la solution la plus adaptée se pose. Le responsable informatique nous expliquait ainsi qu'en matière d'interconnexion, il hésitait entre une prestation d'interconnexion de réseau globale confiée à un opérateur et des solutions plus partielles reposant sur le réseau public de l'internet, et s'appuyant sur le fait que les tarifs de connexion ADSL sont extrêmement bas dans certains pays où le groupe est actif.

\section{Embaltube : un groupe polyvalent en voie d'intégration de ses systèmes d'information}

31 Le groupe Embaltube constitue encore une autre configuration. Il emploie au total environ 400 personnes, dans une demi-douzaine de sociétés de l'ordre de 70 ou 80 personnes chacune, et réparties sur l'ensemble du territoire français. L'emballage est ce qui fait l'unité du groupe, les sociétés qui le composent étant chacune spécialisées sur une variante particulière : l'emballage en carton pour l'une d'entre elle, les flacons à parfum pour une autre, les flacons pour boisson de consommation, etc. Au moment où nous réalisons l'entretien, le groupe-est en train de mettre en place un travail de collaboration entre les responsables des filiales, dont notre interlocuteur nous dira que l'enjeu est «d'éviter la réunionite classique dans ce genre de situations ». Il semble ne pas y avoir de stratégie commerciale commune au sein du groupe. La gestion des ressources en commun est limitée ou balbutiante: aucune centralisation des achats n'est mise en place, mais certains frais communs sont partagés, comme par exemple pour la participation à des expositions. Les différents responsables des filiales semblent former un réseau avec de fortes relations interpersonnelles mettant en place des logiques de coopération qui n'ont pas (encore) un caractère global.

32 Au total, on est dans une situation dans laquelle l'intégration gestionnaire, commerciale, informatique, est en train de se faire : on commence à faire circuler l'information dans le groupe sur les besoins de clients; une mutualisation des ressources se met en place; on communique encore entre sociétés via internet, avec des adresses mail qui ne sont pas nominatives, mais on envisage d'interconnecter les réseaux et de développer un intranet; le site web de l'une des sociétés servira de point de départ pour élaborer le site du groupe. 


\section{Conclusion : vers une analyse des différents modes d'intégration dans les groupements de PME}

Les trois cas que nous avons exposés constituent une très brève introduction à la compréhension des modalités de la communication et de la circulation de l'information dans ces structures complexes et particulière que forment les groupes de PME. D'autres enquêtes seraient nécessaires pour poursuivre ce type de description et pour en tirer un cadre d'analyse. Il nous semble notamment que l'un des enjeux est de comprendre comment s'articulent différentes modalités d'intégration de l'activité collective dans ces structures. L'enquête sur ce petit échantillon nous a permis de repérer plusieurs modalités de l'intégration qui, sur nos trois cas, se positionnent comme suit.

Image3

Tableau 2 : Différentes modalités de l'intégration dans des groupements de PME

L'intégration financière correspond à l'existence de liens entre plusieurs sociétés par l'intermédiaire de participations financières. C'est bien l'existence de ce type d'intégration qui spécifie en première approximation l'existence d'un groupe. Néanmoins, il peut y avoir des situations dites de « grappes » (Debray, 1997) proches des groupes sans toutefois avoir cette structure : c'est le cas lorsqu'une même personne est à la tête de plusieurs sociétés, même lorsqu'il n'existe aucune liaisons financières entre elles. On peut qualifier ce type d'intégration d'entrepreneuriale: c'est bien le même entrepreneur qui assurera le lien entre plusieurs sociétés qui n'entretiennent aucune forme de dépendance croisée. On pourra dire qu'il existe une intégration gestionnaire dès lors que certaines fonctions transverses ont fait l'objet d'une harmonisation (paie, comptabilité, etc). Elle est à différencier d'une intégration plus organisationnelle, qui traduit le fait de l'existence de coopérations entre des équipes appartenant à différentes entreprises du groupe. Dans le cas de Martin Leclair par exemple, il semble qu'il n'y ait aucun lien entre les équipes de designer de luminaires de la filiale Italienne et les équipes de conception de la filiale située à Vitry. Inversement, le travail en projet transverse entre équipes des différentes filiales semble fortement développé à Publi-Factory, ce qui spécifie une forte intégration organisationnelle. L'intégration commerciale va pouvoir se traduire à plusieurs niveaux: partages de fichiers clients, existence d'une stratégie commune, stratégie de marque, etc. Enfin, la notion d'intégration informationnelle vise à traduire le degré d'interconnexion des systèmes d'information, et l'existence d'une démarche commune en matière de communication. Comme on peut le voir sur nos exemples, il semble qu'il n'y ait pas forcément de hiérarchie facilement repérable entre ces différents modes d'intégration. Préciser la façon dont ces modalités s'articulent les unes aux autres constitue sans doute un objectif d'intérêt pour des recherches sur la communication dans les groupes de PME. 


\section{BIBLIOGRAPHIE}

BECATTINI, Giacomo (1992). Le district marshallien : une notion socio-économique. Les régions qui gagnent. Districts et réseaux : les nouveaux paradigmes de la géographie économique. G. Benko and A. Lipietz. Paris, PUF : 35-56.

BOUTARY, Martine (2003). TIC et PME : des usages aux stratégies. Paris, L'Harmattan.

CASTELLS, Manuel (1998). La société en réseaux. Paris, Fayard.

COURAULT, Bruno (2000). « Districts italiens et PME-systèmes français : comparaison n'est pas raison. » La lettre du CEE (61).

COURAULT, Bruno et TROUVE, Philippe (2001). Les dynamiques des PME. Approches internationales. Paris, Cahier du CEE n 38.

CROUCH, Colin, LE GALES, Patrick, TRIGILA, Carlo and VOELZKOW, Helmut (2001). Local production Systems in Europe, Rise or Demise ? Oxford, Oxford University Press.

DEBRAY, Caroline (1997). Structures et stratégies de l'hypogroupe. Sciences de gestion. Montpellier, Université de Montpellier 1.

DEBRAY, Caroline, LEYRONAS, Christophe (1998). Réseau et hypogroupe : l'émergence de pratiques managériales en PME. PME - De nouvelles approches. O. Torres. Paris, Economica. 83-93.

DEBRAY-BOUVAREL, Caroline (2000). Contrôles dans l'hypogroupe : implications organisalionuelles et managériales d'une stratégie de croissance en PME. Communication à la IXème conférence internationale de management stratégique, AIMS, Montpellier, 24-26 mai 2000.

DHALEINE, Laurence, LICOPPE, Christian and MALLARD, Alexandre (2001). NTIC et Internet dans les PME-PMI. Premiers éléments pour une typologie des usages. Comprendre les usages d'Internet. E. Guichard. Paris, Edition Rue d'Ulm. Presses de l'Ecole Normale Supérieure : 43-51.

FEUVRIER, Paul (2000). « Les groupes structurent l'industrie française. » SESSI, Le 4 pages des statistiques industrielles (127).

GADILLE, Martine and D'IRIBARNE, Alain (2000). « La diffusion d'Internet dans les PME : motifs d'adoption, réseaux et ressources mobilisées. » Réseaux (104) : 61-92.

HARISSON, Bennet (1995). Lean and Mean, Guilford Press.

INSEE (2004). Retour d'information - Enquête sur les liaisons financières. INSEE.

INSEE méthodes (1997). « Les réseaux d'entreprises : des collectifs singuliers. » (67-68).

JACQUEMIN, Alexis (1989). « La dynamique du groupe d'entreprises : une perspective de droit économique. » Revue d'Economie Industrielle (47) : 6-13.

JULIEN, Pierre-André, MARCHESNAY, Michel (1996). L'entrepreneuriat. Paris, Economica.

LAUBACHER, Robert J. and MALONE, Thomas W. (2001). Two scenarios for 21st Century Organisations : Shifting Networks of Small Firms or All-Encompassing « Virtual Countries ». Cambridge, MA, Sloan School of Management, MIT. 
LOISEAU, Hervé (2001). « Des groupes de la taille d'une PME. Un phénomène en plein essor. » Insee Première (764).

MARCHESNAY, Michel (1991). « De l'hypofirme à l'hypogroupe : naissance, connaissance, reconnaissance. » Les cahiers du Lerass (23).

MARSCHENAY, Michel (2003). « La petite entreprise : sortir de l'ignorance. » Revue Française de Gestion vol. 29(144) : 108-118.

MENGUZZATO-BOULARD, Martina, ESCRIBA-ESTEVE, Alejandro and SANCHEZ-PENAIDO, Luz (2003). Les accords de coopération : une stratégie pour toutes les entreprises ? Communication à la XIIème conférence de l'Association Internationale de Management Stratégique, Carthage, AIMS.

MOATI, Philippe (2002). L'entreprise du xxi ${ }^{\mathrm{e}}$ siècle. Paris, Editions de l'aube - Datar.

PIORE, M.J. and SABEL, CHARLES F. (1984). Les chemins de la prospérité. De la production de masse à la spécialisation souple.

PUTHOD, Dominique (1998). L'alliance, une option stratégique permettant de contourner les dilemmes classiques de la PME. PME - De nouvelles approches. O. Torres. Paris, Economica.

SKALITZ, Anne (2002). « Au delà des entreprises : les groupes. » Insee Première (836).

TORRES, Olivier (1998). PME - de nouvelles approches. Paris, Economica.

TROUVE, Philippe and COURAULT, Bruno (2000). Des métamorphoses de la petite entreprise aux dynamiques des PME. Exploration du cas Français. Les dynamiques de PME. Approches internationales. P. Trouvé and B. Courault. Paris, PUF : xvi-lxxix.

TROUVE, Philippe and COURAULT, Bruno, Eds. (2000). Les dynamiques des PME. Approches internationales. Paris.

VERGEAU, E., CHABANAS, N. (1997). « Le nombre de groupes d'entreprises a explosé en 15 ans. » Insee Première (553).

\section{NOTES}

1. Courault et Trouvé signalent également deux autres ligures plus minoritaires dans le paysage industriel de cette époque: d'une part les pentes entreprises qui ont incarné des tentatives utopiques d'alternative au modèle social et économique dominant («usines à la campagne, développement autocentré, entreprises alternatives.. »); d'autre part les unités organisées en districts industriels (dans le contexte italien) ou en systèmes productifs localisés (dans le contexte français).

2. Trouvé et Courault rappellent en effet les travaux de Boccara qui tempèrent considérablement la croyante commune en l'efficacité des PME dans la création d'emploi : «les travaux les plus récents établissent que ce sont les PME évoluant «dans le contour des groupes » qui créent ou maintiennent le plus d'emplois (Boccara, $1997 ; 1998)$ et que parmi les plus petites, ce sont celles qui créent le plus d'emplois qui en détruisent aussi le plus (enquête DMMO/EMMO du ministère du travail). En dépit des certitudes de la pensée commune, et à cause de la complexité de la reconfiguration actuelle des systèmes productifs, il est désormais devenu quasiment impossible de cerner scientifiquement la contribution spécifique des PMF. à l'emploi » (Trouvé et Courault, 2000, p XLVIII)

3. Comme le précise Hervé Loiseau (2001), telle enquête ne "voit " pas toutes les entreprises, puisqu'elle interroge celles qui disposent d'un portefeuille de plus de 8 millions de F de titres de 
participations, ou qui emploient plus de 500 salaries ou réalisent un chiffre d'affaire supérieur à 200 millions de francs. Elle sous-estime donc le nombre de groupes. Une estimation réalisée sur une région française suggère qu'elle détecte environ les $2 / 3$ des entreprises dépendant d'un groupe. Celles qui échappent à l'enquête emploient environ 25 salariés, contre 71 pour les autres. 4. Cette étude a été réalisée avec Alexandre Largier, pour le compte de Didier Guy à la Direction de l'Innovation et Marie-Cécile Helleboid à France Télécom R\&D, que nous tenons à remercier ici pour leur intérêt vis-à-vis de ce travail.

5. Bien entendu, les noms et les lieux cités dans toute cette partie sont fictifs, de façon à garantir l'anonymat des firmes.

\section{RÉSUMÉS}

Un nombre croissant de PME tendent aujourd'hui à se structurer dans des groupes. Cet article s'interroge sur la nature de ce phénomène de concentration capitalistique et financière dans le secteur des petites entreprises. Pour cela, il resitue le phénomène des groupes de PME dans l'histoire industrielle récente, notamment du point de vue des relations que ces entités entretiennent avec les grandes entreprises et les PME indépendantes. Il rappelle les données statistiques et d'analyse stratégique disponibles pour le comprendre. Il propose pour terminer quelques éléments issus d'une enquête qualitative, permettant d'introduire à une compréhension des modalités de la communication dans les groupes de PME.

À growing number of SMEs structures their activity in groups. This article questions the nature of this phenomenon of capital intensive and financial concentration observable in the sector of the small companies. It contextualises the process of group formation for SMEs in the recent industrial history, taking into account the relations between these entities and the large companies and independent SMEs. Il recalls the statistical data and strategic analysis approaches available to describe the process. Eventually, it outlines some empirical finding drawn front a qualitative survey, which introduce to the understanding of the modalities of communication inside the groups of SMEs.

\section{AUTEUR}

\section{ALEXANDRE MALLARD}

Sociologue, responsable de l'unité de recherche et développement sur les Usages des TIC dans les Entreprises (UTE) au laboratoire « Sociologie des Usages et Traitement Statistique de l'Information » de la division R\&D de France Télécom. Ses recherches portent sur l'appropriation et les usages des nouvelles technologies dans les petites et très petites entreprises. 